\title{
Investigation of injection dose and camera integration time on quantifying pharmacokinetics of a Cy5.5-GX1 probe with dynamic fluorescence imaging in vivo
}

\author{
Yunpeng Dai \\ Xueli Chen \\ Jipeng Yin \\ Xiaoyu Kang \\ Guodong Wang \\ Xianghan Zhang \\ Yongzhan Nie \\ Kaichun $\mathrm{Wu}$ \\ Jimin Liang
}




\title{
Investigation of injection dose and camera integration time on quantifying pharmacokinetics of a Cy5.5-GX1 probe with dynamic fluorescence imaging in vivo
}

\author{
Yunpeng Dai, ${ }^{a}$ Xueli Chen, ${ }^{a, \star}$ Jipeng Yin, ${ }^{b}$ Xiaoyu Kang, ${ }^{b}$ Guodong Wang, ${ }^{b}$ Xianghan Zhang, ${ }^{a}$ Yongzhan Nie, \\ Kaichun Wu, and Jimin Liang ${ }^{\mathrm{a}, *}$ \\ ${ }^{a}$ Xidian University, Engineering Research Center of Molecular and Neuro Imaging of Ministry of Education and School of Life Science and \\ Technology, 266 Xinglong Section of Xifeng Road, Xi'an 710071, China \\ ${ }^{\mathrm{b}}$ Fourth Military Medical University, State Key Laboratory of Cancer Biology and Xijing Hospital of Digestive Diseases, Xijing Hospital, \\ 127 Changle Road, X'an 710032, China
}

\begin{abstract}
The aim of this article is to investigate the influence of a tracer injection dose (ID) and camera integration time (IT) on quantifying pharmacokinetics of Cy5.5-GX1 in gastric cancer BGC-823 cell xenografted mice. Based on three factors, including whether or not to inject free GX1, the ID of Cy5.5-GX1, and the camera IT, 32 mice were randomly divided into eight groups and received 60-min dynamic fluorescence imaging. Gurfinkel exponential model (GEXPM) and Lammertsma simplified reference tissue model (SRTM) combined with a singular value decomposition analysis were used to quantitatively analyze the acquired dynamic fluorescent images. The binding potential (Bp) and the sum of the pharmacokinetic rate constants (SKRC) of Cy5.5GX1 were determined by the SRTM and EXPM, respectively. In the tumor region, the SKRC value exhibited an obvious trend with change in the tracer ID, but the Bp value was not sensitive to it. Both the Bp and SKRC values were independent of the camera IT. In addition, the ratio of the tumor-to-muscle region was correlated with the camera IT but was independent of the tracer ID. Dynamic fluorescence imaging in conjunction with a kinetic analysis may provide more quantitative information than static fluorescence imaging, especially for a priori information on the optimal ID of targeted probes for individual therapy. $\odot 2016$ Society of Photo-Optical Instrumentation Engineers (SPIE) [DOI: 10.1117/1.JBO.21.8.086001]
\end{abstract}

Keywords: dynamic fluorescence imaging; dose dependency; pharmacokinetic quantification; Cy5.5-GX1.

Paper 160226R received Apr. 9, 2016; accepted for publication Jul. 14, 2016; published online Aug. 3, 2016.

\section{Introduction}

Gastric cancer is a common malignant tumor, which is one of the most frequent causes of cancer-related death, especially in developing countries. ${ }^{1,2}$ The conventional treatment includes surgery, radiation therapy, and chemotherapy. However, gastric cancer is difficult to cure unless it can be detected at an early stage. $^{3,4}$ Therefore, the treatment of gastric cancer needs a unique treatment approach; fortunately, angiogenesis-targeted therapy has emerged as a promising approach for it. ${ }^{5}$ Correspondingly, a cyclic 9-mer peptide named GX1 was identified by Zhi et al., ${ }^{6}$ which exhibits the specific ability to target vasculature of gastric cancer. ${ }^{7-9}$ Furthermore, the GX1 peptide was successfully conjugated with near-infrared (NIR) fluorescent dye Cy5.5 to obtain the Cy5.5-GX1 probe, which could be used for tumor-targeted imaging and detection. ${ }^{10}$ Unfortunately, the in vivo pharmacokinetic properties of GX1 have not been fully studied yet. ${ }^{11}$ The injection dose (ID) of the GX1-based probe used in current studies is often determined according to experience, and the optimal dose is also unexplored. Appropriate ID of targeted probes not only avoids receptor oversaturation and wasting of probes but also can avoid the phenomenon of image overexposure caused by excessive probe injections. Thus, extensive investigations focused on the influence of the probe ID on pharmacokinetics quantification may provide valuable information about the optimal dose of

*Address all correspondence to: Xueli Chen, E-mail: xlchen@xidian.edu.cn; Jimin Liang, E-mail: jimleung@ @ail.xidian.edu.cn molecular therapeutic agents for preclinical trials. On the other hand, camera integration time (IT) is also an important parameter that can affect the quality of dynamic fluorescence imaging. ${ }^{12}$ Therefore, it is necessary to do further in-depth research on the tracer ID and camera IT-dependent pharmacokinetic quantification, which would be valuable for the preclinical application of GX1-based targeted probes.

Because it can provide functional information at cellular and molecular levels for image-guided optimization and management of personalized cancer treatment, noninvasive molecular imaging of angiogenesis may be a method for the early diagnosis of cancer. ${ }^{13,14}$ Over the past few decades, NIR fluorescence imaging has attracted more and more attention because of its nonradiation property and low tissue absorption. ${ }^{15-19}$ In particular, dynamic fluorescence imaging combined with a kinetics analysis offers multiple advantages in comparison to static images. For example, it can estimate the kinetic parameters, which can be used to calculate the metabolic rate and receptor density. ${ }^{20,21}$ Kinetic analysis can also separate a specific signal from a nonspecific one, which further improves the accuracy of measuring the binding potential $(\mathrm{Bp})$ of a targeted probe. ${ }^{22-24}$ Some in vivo quantitative studies have also been carried out by dynamic fluorescence imaging combined with the kinetics analysis. ${ }^{25-30}$ As described in Ref. 28, using dynamic fluorescence imaging together with an exponential model, Kwon et al. successfully determined the uptake parameters as

$1083-3668 / 2016 / \$ 25.00$ @ 2016 SPIE 
a function of the ID of Cy5.5-c(KRGDf) in the tumor region. $\mathrm{Zhu}$ et al. ${ }^{30}$ designed and synthesized a $64 \mathrm{Cu} / \mathrm{ZW}-1$-based dual-modality imaging probe [c(RGDyK)-C(DOTA)-ZW-1] to demonstrate for the first time that dynamic fluorescence imaging could obtain comparable quantitation results in vivo compared with dynamic positron emission tomography (PET) imaging. In addition, in our previous study, we quantified molecular specificity of Cy5.5-labeled cyclic 9-mer peptide (Cy5.5-GX1) in vivo with dynamic fluorescence imaging, which provides better understanding of its kinetic properties. ${ }^{11}$

In this study, we investigated the influence of the tracer ID and camera IT on quantifying pharmacokinetics of Cy5.5-GX1 in gastric cancer-bearing nude mice. Based on three factors, including whether or not to inject free GX1, the ID of Cy5.5-GX1, and the camera IT, 32 mice were randomly subdivided into eight test groups and received 60-min dynamic fluorescence imaging. We used the Gurfinkel exponential model (GEXPM) and Lammertsma simplified reference tissue model (SRTM) combined with the singular value decomposition (SVD) analysis to quantitatively analyze the obtained dynamic fluorescent images. Here, the SRTM was selected to estimate the Bp of the injected tracer. The $\mathrm{Bp}$ is one of the most important macroparameters for pharmacokinetic quantification of Cy5.5-GX1, which is defined as the product of tracer binding affinity and available receptor density. ${ }^{30-32}$ Correspondingly, GEXPM was selected to calculate the sum of the pharmacokinetic rate constants (SKRC), which is another important macroparameter that reflects receptor-ligand affinity. ${ }^{29}$ In addition, the reason for choosing these two models is because they are able to avoid blood sampling and capture the parameters of interest. ${ }^{33}$ Then, the change of Bp and SKRC as a function of the tracer ID and camera IT was drawn upon, whose results would provide valuable references for preclinical applications of the GX1 probe.

\section{Materials and Methods}

\subsection{Cy5.5-GX1 Synthesis and Immunofluorescent Staining}

Cy5.5-GX1 was synthesized by FanBo Co Ltd. (Beijing, China) and used after identification with high-performance liquid chromatography (HPLC) and time-of-flight mass spectrometry (TOFMS). The peptide was saved at $-20^{\circ} \mathrm{C}$ after the freeze-drying process. In addition, immunofluorescent staining and related results are described in Ref. 11.

\subsection{Tumor Model Construction}

All procedures for the animal study were performed in accordance with the Fourth Military Medical University (FMMU) animal protocol. All athymic female nude BALB/c mice (4 to 6 weeks; about $22 \mathrm{~g}$ ) were provided by the Laboratory Animal Center, FMMU. The BGC-823 cancer cells were harvested during the logarithmic growth phase and suspended in phosphate buffer saline $\left(1 \times 10^{7}\right.$ cells $\left./ \mathrm{ml}\right)$. Approximately, a 200- $\mu$ l cell suspension was injected into the right shoulder subcutaneously in each mouse (about $2 \times 10^{6}$ cells/mouse). The tumor volume was measured with calipers three times a week, until it reached about $300 \mathrm{~mm}^{3} .^{30}$

\subsection{Dynamic Fluorescence Imaging and Regions of Interest Extraction}

Data acquisition for dynamic fluorescence imaging was completed by the IVIS kinetic imaging system from Caliper Life Sciences (PerkinElmer, Hopkinton, Massachusetts). A laser source of $660 \mathrm{~nm}$ was used to excite the fluorophore, and a bandpass filter centered on $710 \mathrm{~nm}$ was used for the collection of the fluorescence emission. Based on three factors, including whether or not to inject the free GX1, the ID of Cy5.5-GX1, and the camera IT, 32 mice were randomly divided into eight groups $(N=4)$. The detailed description of grouping is summarized in Table 1, wherein the fifth group (termed as the blocked group) received an excess of free GX1 peptide (about $500 \mathrm{nmol}$ ) for $1 \mathrm{~h}$ before the tracer injection. During the tracer injection and dynamic fluorescent data acquisition, isoflurane and a thermostat-controlled thermal heater were used to anesthetize mice and maintain body temperature, respectively. Five background images were collected prior to the tracer injection. BGC-823 bearing mice were subjected to dynamic fluorescence imaging for $1 \mathrm{~h}$ immediately after the probe intravenous injection and the frame rates were set to be $90 \times 6 \mathrm{~s}, 75 \times 12 \mathrm{~s}$, and $36 \times 60 \mathrm{~s}$. Moreover, fluorescent images from each group were acquired again after $24 \mathrm{~h}$ of postinitial injection.

Regions of interest (ROIs) extraction and dynamic fluorescent data processing were conducted with Living Image 4.4 software and MATLAB ${ }^{\circledR}$ 2014b (The MathWorks, Inc., Natick, Massachusetts), respectively. For the quantitative analysis, tumor ROI and muscle ROI were both determined in the white light image, with the same surface area. The muscle ROI was the region of muscle contralateral to the tumor, where there is a region with no specific binding that acts as a reference tissue. ${ }^{10}$ When we subtracted the average of the background images from the fluorescent images, the mean of the fluorescent signals $\left(\times 10^{6}\right.$ photon $\left./ \mathrm{cm}^{2} / \mathrm{s}\right)$ within each ROI was calculated. Thereafter, the fluorescence time-activity curves (TACs) of each ROI could be determined.

\subsection{Kinetic Models and Singular Value Decomposition Analysis}

We used a two-tissue (three-compartment) model to describe the GX1 tracer kinetics in the tumor region, which assumes that three possible environments (compartments) exist for GX1. They include unmetabolized tracer in the blood plasma compartment, free and nonspecific binding tracer in the extravascular and intracellular space, and tracer specifically bound to the GX1 receptor. ${ }^{34}$ In the reference region, a one-tissue (two-compartment) model was employed to describe the GX1 tracer kinetics. The Bp and the SKRC of Cy5.5-GX1 were determined

Table 1 Detailed description of grouping in this study.

\begin{tabular}{lcccccccc}
\hline Groups & & & & & & & \\
& 1 & 2 & 3 & 4 & 5 & 6 & 7 \\
\hline ID nmol (mg/kg) & $0.5(43.18)$ & $1(86.36)$ & $1(86.36)$ & $1(86.36)$ & $1(86.36)$ & $1.5(129.54)$ & $2(172.72)$ & $3(259.08)$ \\
IT (s) & 1 & 0.5 & 1 & 1.5 & 1 & 1 & 1 & 1 \\
\hline
\end{tabular}


by the SRTM and the EXPM, respectively. A detailed description of kinetic models and related parameters can be found in a previous study. ${ }^{11}$

Before applying the SRTM, we processed the raw fluorescent data with the SVD analysis to unmix the specific signal component in the tumor region. ${ }^{11,30}$ The SVD analysis was performed on the two-dimensional spatial-temporal matrices formed by the TACs' background, muscle, and tumor ROIs to obtain a series of eigenvalues and eigenvectors. The L-curve method was used to threshold the eigenvalues, separating the meaningful eigenvectors. There was one signal component in the background ROI (corresponding to autofluorescence), two signal components in the muscle ROI (corresponding to autofluorescence and muscle uptake), and three signal components in the tumor ROI (corresponding to autofluorescence, muscle uptake, and tumor-specific uptake). Finally, the pure tumor and muscle TACs could be recovered by projecting the raw muscle and tumor TACs onto the corresponding identified signal components.

\subsection{Statistical Analysis}

Pharmacokinetic parameters quantitatively estimated from the dynamic fluorescence data were expressed as their mean values together with the standard deviation (means $\pm \mathrm{SD}, N=4$ ). SPSS software (Version 22.0 for Windows, Chicago, USA) was employed to conduct all of the statistical analyses. Statistical significance was evaluated using a one-way ANOVA and a student's $t$-test, and a least significant difference (LSD) test was used to control type II errors and carry out posthoc testing to confirm pairwise significance. $P$ values smaller than 0.05 were considered to be statistically significant.

\section{Results}

\subsection{Dynamic Fluorescence Imaging}

Figure 1(a) shows one of the representative raw fluorescent images (without background subtraction) at $24 \mathrm{~h}$ after the 2-nmol Cy5.5-GX1 injection. The tumor could be identified from the fluorescent images in the process of data acquisition. To confirm the specificity of Cy5.5-GX1 in vivo, dynamic fluorescence imaging was performed on the mice, which received an excess of free GX1 peptide as a blocked agent $1 \mathrm{~h}$ prior to the Cy5.5-GX1 injection. The corresponding representative raw fluorescent image is shown in Fig. 1(b). Under the same conditions of camera IT and dynamic display scale, there were no obvious fluorescent signals in the tumor region for the blocked experiment. Especially in the fluorescent image at $24 \mathrm{~h}$ after the probe injection, almost no fluorescent signal was observed in the tumor region. The high-intensity fluorescent signal in the kidneys suggested that Cy5.5-GX1 was excreted through the bladder. ${ }^{35}$

Figure 2(a) shows the tumor-to-muscle region (TMR) ratio as a function of time after the tracer injection for the experiments with the same tracer ID but different camera ITs. We found that the longer the camera IT, the greater the TMR value. Especially for the time point at $24 \mathrm{~h}$ after the tracer injection, there was a significant statistical difference in the TMR for the three groups $(p<0.01$, ANOVA). Figure 2(b) shows the TMR as a function of lapse time after the tracer injection for another four selected groups. Three groups were selected with the same camera IT but different tracer IDs, whose posthoc testing results showed that their TMRs had no statistical difference. The remaining group was the blocked group (ID: $1 \mathrm{nmol}$, IT: $1 \mathrm{~s}$ ) and the TMR value was significantly different from that of the former three groups (all $p<0.01$, LSD).

\subsection{Kinetic Analysis}

First, we analyzed the Bp value as a function of the camera IT and tracer ID. For the investigation of the camera IT, we used the same tracer ID but different camera ITs. The estimated Bp values are shown in Fig. 3(a). Statistical analysis demonstrated that the $\mathrm{Bp}$ values in this investigation experiment had no statistical significance $(p=0.81$, ANOVA). In the investigation of the tracer ID, the camera IT was fixed at $1 \mathrm{~s}$, but the tracer ID was set to be from 0.5 to $3 \mathrm{nmol}$. In addition, the blocked

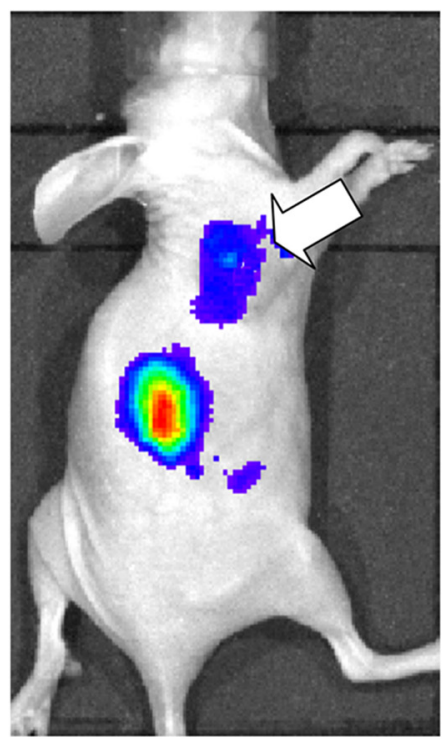

(a)

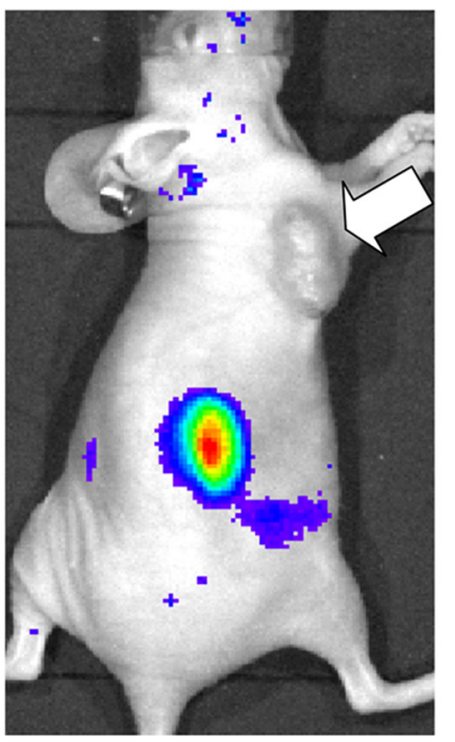

Epifluorescence

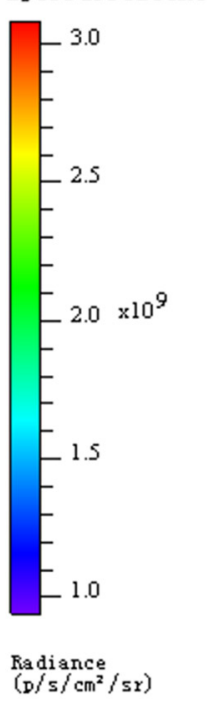

(b)

Fig. 1 Representative raw fluorescent images (without background subtraction) generated at $24 \mathrm{~h}$ after administration of (a) 1-nmol Cy5.5-GX1 injected alone and (b) excessive free GX1 injected $1 \mathrm{~h}$ prior to the Cy5.5-GX1 injection. White arrows indicate the tumor locations. 


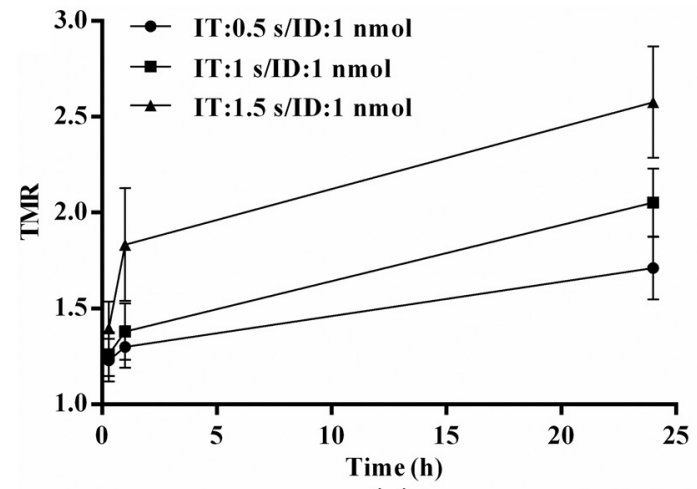

(a)

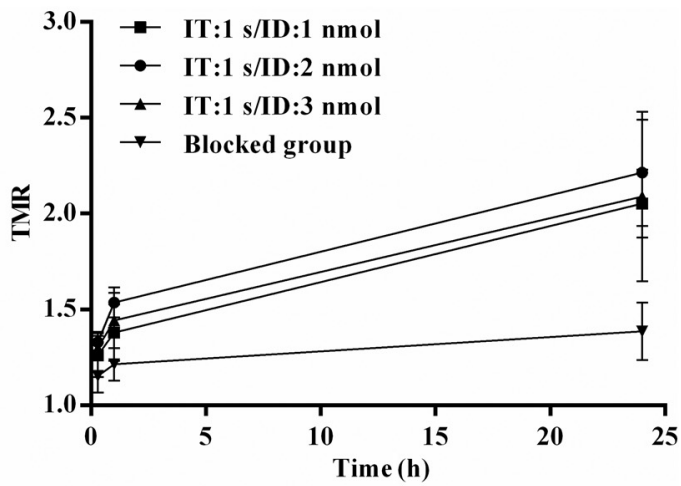

(b)

Fig. 2 Ratio of the TMR as a function of time after the tracer injection: (a) groups with the same tracer ID but different camera ITs and (b) three groups with the same IT but different tracer IDs, and a blocked group.

experiments were also conducted. Figure 3(b) shows the estimated $\mathrm{Bp}$ values for this investigation experiment. Posthoc testing results confirmed that there was also no statistical difference for the Bp values in different ID groups. However, if excessive amounts of free GX1 were injected as a blocking agent, the corresponding $\mathrm{Bp}$ values were significantly different from the others (all $p<0.01$, LSD).

Second, we analyzed the relationship of the SKRC values in the tumor and muscle ROIs with the tracer ID and camera IT. In the first step, we fixed the tracer ID as $1 \mathrm{nmol}$ and changed the camera IT to $0.5,1$, and $1.5 \mathrm{~s}$. Figure 3(c) shows the estimated SKRC values in the tumor and muscle ROIs, respectively. We found that the SKRC values exhibited no statistical difference for different camera ITs wherever they were (in the tumor ROIs: $p=0.52$ and in the muscle ROIs: $p=0.50$, ANOVA). In the second step, the camera IT was fixed at $1 \mathrm{~s}$, but the tracer ID was changed from 0.5 to 3 nmol. Similarly, the blocked group that was injected with excessive amounts of free GX1 was also included. Figure 3(d) shows the related statistical analysis results. From Fig. 3(d), some interesting conclusion could be addressed. First, an approximate linear relationship between the SKRC values estimated in the tumor ROIs and the Cy5.5-GX1 ID was observed from 0.5 to $1.5 \mathrm{nmol}$. The SKRC value became larger as the tracer ID increased. Second, the SKRC values estimated in the tumor ROIs remained unchanged when the tracer ID ranged from 1.5 to $3 \mathrm{nmol}$, and posthoc testing results indicated no statistical difference between them. However, they were statistically different from

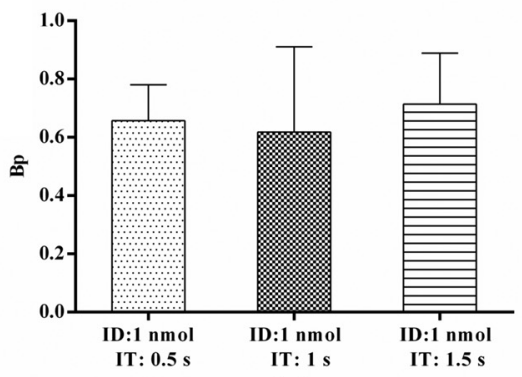

(a)

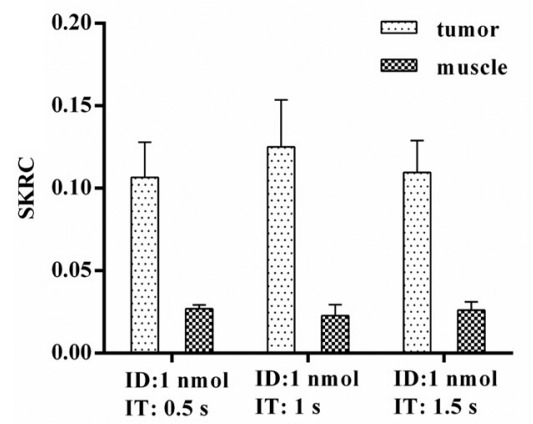

(c)

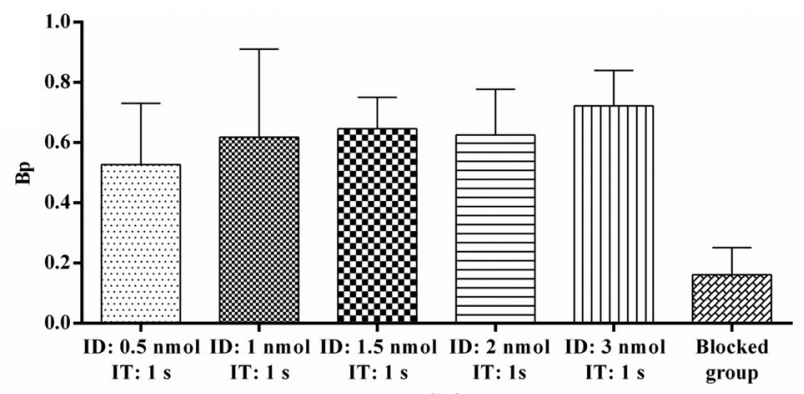

(b)

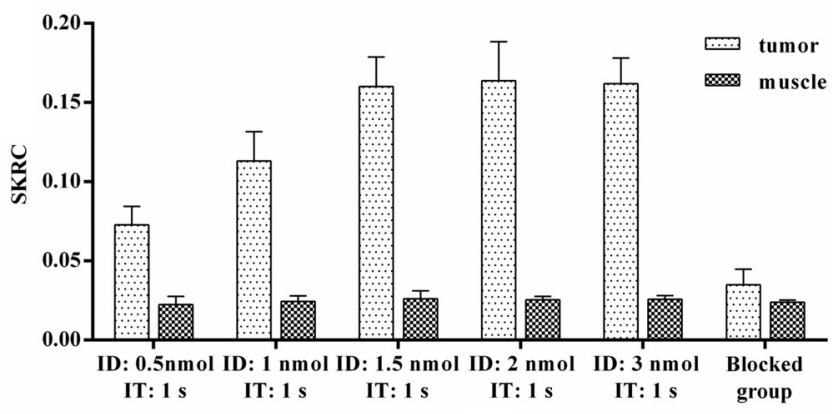

(d)

Fig. 3 Bp values of Cy5.5-GX1 and SKRC values estimated in the tumor and muscle ROls as a function of the camera IT and tracer ID, respectively. Relationships between (a) Bp values and different camera ITs, (b) Bp values and different tracer IDs, (c) SKRC values and different camera ITs, and (d) SKRC values and different tracer IDs. 
those with a 0.5 - and 1-nmol tracer ID (all $p<0.01$, LSD). Third, the SKRC values estimated in the muscle ROIs did not show any statistical difference for different tracer IDs $(p=0.53$, ANOVA). Lastly, in the blocked group, there was no statistical difference for the SKRC values between the tumor and muscle ROIs ( $p=0.08, t$-test). However, the SKRC values of tumor ROIs in the blocked group were significantly different from those estimated in other nonblocked groups (all $p<0.01, \mathrm{LSD}$ ).

\section{Discussion}

Angiogenesis is the basis of tumor progression, so vascular suppression therapy is a promising remedy that can bring new hope to cancer patients. ${ }^{36}$ The identification of molecules with receptor binding affinity is essential for this treatment. It can promote the specific targeting ability of agents, which can further improve the therapeutic effect. In this study, with the help of dynamic fluorescence imaging, we observed an increase and retention of Cy5.5-GX1 uptake in tumor ROIs. Such a specific uptake was abrogated when excess amounts of free GX1 peptide were injected $1 \mathrm{~h}$ before the tracer injection. These results demonstrated that Cy5.5-GX1 was a promising probe with receptor binding affinity in vivo and had great potential in application of vascular suppression therapy. In Sec. 3.1, statistical analysis results suggested that the TMR of Cy5.5-GX1 was dependent on the camera IT but was not sensitive to the tracer ID. In other words, we can increase the camera IT to obtain a higher TMR value.

Compared with conventional static imaging, dynamic fluorescence imaging combined with kinetic analysis provides more information on Cy5.5-GX1 in the metabolic process, which may accelerate the clinical application of GX1. One of the most interesting parameters is $\mathrm{Bp}$, which is used to estimate the available receptor density in the ROIs and is also considered as an indicator of the equilibrium state of the tracer. ${ }^{34}$ From Fig. 3(b), we did not find any significant difference for Bp values when the ID of Cy5.5-GX1 was increased from 0.5 to $3 \mathrm{nmol}$, indicating that the available receptor of GX1 did not reach its saturation state, which was in this dose range. Similarly, no statistical difference was observed for the SKRC values in the tumor ROIs when the ID of Cy5.5-GX1 was increased from 1.5 to $3 \mathrm{nmol}$. Such a dose-independent response may be caused by the mechanism of partial self-inhibition at a higher dose rather than saturated receptor binding. ${ }^{28,37}$ This is because the SKRC value is equal to the sum of the kinetic parameters, which cannot be used directly to estimate the available receptor density. Because GEXPM describes the kinetic characteristics of the tracer with a one-way process that counts only its continuous accumulation in the tumor ROI, it may be more appropriate to reflect on the kinetics of the tracer during the initial stage of the injection. As a result, the combination of SKRC and Bp values may be more comprehensive in describing the kinetic characteristics of the tracer in vivo.

If we fixed the tracer ID and changed the camera IT, there would be no statistical differences observed for both the SKRC and $B p$ values. This suggests that the SKRC and Bp values were independent of the camera IT. This conclusion is useful when we want to obtain a high TMR value without compromising the dynamic quantitative results. At a lower ID $(0.5 \mathrm{nmol})$, the tracer may selectively accumulate in the kidneys or other parts rather than in the tumor region, which may reduce the ratio of tumorto-muscle. ${ }^{38}$ The resulting Bp values and SKRC values of tumor
ROIs are lower, but both of them are still significantly different from those of the blocked group ( $p<0.05$, LSD). In addition, when the tracer ID reaches $6 \mathrm{nmol}$ and the camera IT is set at $0.5 \mathrm{~s}$, saturated pixels will appear in the acquired fluorescent images. In this case, one needs to reduce the camera IT to avoid saturated pixels, but this also leads to a decrease in TMR. From this point of view, a higher tracer ID may not be suitable for the quantitative analysis. In conclusion, the $\mathrm{Bp}$ and SKRC are essential kinetic parameters when using dynamic fluorescence imaging for the quantitative analysis, and both of them can provide more information on ligandreceptor binding than the TMR obtained from a static fluorescent image.

In this study, we evaluated the influence of the tracer ID and camera IT for quantifying the pharmacokinetics of Cy5.5-GX1. We also compared the performance of the pharmacokinetic parameters obtained by dynamic fluorescence imaging with the TMR obtained from a static fluorescent image in the quantitative analysis. In dynamic fluorescence imaging, the determination of the targeted probe ID could avoid receptor oversaturation and wasting of probes, which makes the obtained quantitative results more accurate and reliable. Hence, our quantitative results may provide valuable information about the optimal dose of molecular therapeutic agents for the preclinical application of GX1-based targeted probes, such as the preclinical study of individual therapy. In addition, the camera IT is also a quantitative factor, which has to be considered, and it is related to the quality of dynamic fluorescence imaging. The determination of the relationship between camera IT and pharmacokinetic parameters is beneficial for a more reasonable design of the experimental program. In summary, our quantitative methods and results will provide a valuable reference for future research of GX1-based targeted probes.

\section{Conclusions}

Dynamic fluorescence imaging combined with a kinetic analysis may provide more robust quantitative results than the static fluorescence imaging technique. In particular, it could provide information about the optimal ID for individualized cancer therapy. Our results could provide a reference for future pharmacokinetic studies of GX1 and also promote its future clinical applications.

\section{Acknowledgments}

This work was supported by the National Natural Science Foundation of China under Grant Nos. 81227901, 81571725, 81530058, and 81230033, the Natural Science Basic Research Plan in Shaanxi Province of China under Grant Nos. 2015JQ6249 and 2015JZ019, and the Fundamental Research Funds for Central Universities (Grant Nos. NSIZ021402 and NSIY061406).

\section{References}

1. P. Bertuccio et al., "Recent patterns in gastric cancer: a global overview," Int. J. Cancer 125(3), 666-673 (2009).

2. W. Chen et al., "Cancer statistics in China, 2015," CA: Cancer J. Clin. 66(2), 115-132 (2016).

3. J. L. Pretz et al., "Chemoradiation therapy: localized esophageal, gastric, and pancreatic cancer," Surg. Oncol. Clin. N. Am. 22(3), 511-524 (2013).

4. R. Wadhwa et al., "Modern oncological approaches to gastric adenocarcinoma," Gastroenterol. Clin. N. Am. 42(2), 359-369 (2013).

5. M. V. Backer and J. M. Backer, "Imaging key biomarkers of tumor angiogenesis," Theranostics 2(5), 502-515 (2012). 
6. M. Zhi et al., "Characterization of a specific phage-displayed peptide binding to vasculature of human gastric cancer," Cancer Biol. Ther. 3(12), 1232-1235 (2004).

7. S. Cao et al., "Expression, purification, and characterization of recombinant protein GX1-rmhTNFo," Mol. Biotechnol. 43(1), 1-7 (2009).

8. B. Chen et al., "A novel peptide (GX1) homing to gastric cancer vasculature inhibits angiogenesis and cooperates with TNF alpha in anti-tumor therapy," BMC Cell Biol. 10(1), 63 (2009).

9. X. Hui et al., "Specific targeting of the vasculature of gastric cancer by a new tumor-homing peptide CGNSNPKSC," J. Controlled Release 131(2), 86-93 (2008).

10. K. Chen et al., "A Cy5.5-labeled phage-displayed peptide probe for near-infrared fluorescence imaging of tumor vasculature in living mice," Amino Acids 42(4), 1329-1337 (2012).

11. Y. Dai et al., "In vivo quantifying molecular specificity of Cy5.5-labeled cyclic 9-mer peptide probe with dynamic fluorescence imaging," Biomed. Opt. Express 7(4), 1149-1159 (2016).

12. K. Hwang et al., "Improved excitation light rejection enhances smallanimal fluorescent optical imaging," Mol. Imaging 4(3), 194-204 (2005).

13. S. M. Weis and D. A. Cheresh, "Tumor angiogenesis: molecular pathways and therapeutic targets," Nat. Med. 17(11), 1359-1370 (2011).

14. I. Dimova, G. Popivanov, and V. Djonov, "Angiogenesis in cancer-general pathways and their therapeutic implications," J. BUON 19(1), 1521 (2014).

15. J. Zhang et al., "Incorporating MRI structural information into bioluminescence tomography: system, heterogeneous reconstruction and in vivo quantification," Biomed. Opt. Express 5(6), 1861-1876 (2014).

16. W. Cai and X. Chen, "Multimodality molecular imaging of tumor angiogenesis," J. Nucl. Med. 49(Suppl. 2), 113S-128S (2008).

17. J. V. Frangioni, "In vivo near-infrared fluorescence imaging," Curr. Opin. Chem. Biol. 7(5), 626-634 (2003).

18. S. L. Jacques, "Optical properties of biological tissues: a review," Phys. Med. Biol. 58(11), R37 (2013).

19. S. Luo et al., "A review of NIR dyes in cancer targeting and imaging," Biomaterials 32(29), 7127-7138 (2011).

20. P. Dupont and J. Warwick, "Kinetic modelling in small animal imaging with PET," Methods 48(2), 98-103 (2009).

21. G. Tomasi, F. Turkheimer, and E. Aboagye, "Importance of quantification for the analysis of PET data in oncology: review of current methods and trends for the future," Mol. Imaging Biol. 14(2), 131-146 (2012).

22. N. Guo et al., "Quantitative analysis and parametric imaging of $18 \mathrm{~F}-$ labeled monomeric and dimeric RGD peptides using compartment model," Mol. Imaging Biol. 14(6), 743-752 (2012).

23. N. Guo et al., "Quantitative analysis and comparison study of [18F]AlFNOTA-PRGD2, [18F]FPPRGD2 and [68Ga]Ga-NOTA-PRGD2 using a reference tissue model," PLoS One 7(5), e37506 (2012).
24. K. Shoghi, "Quantitative small animal PET," Q. J. Nucl. Med. Mol. Imaging 53(4), 365-373 (2009).

25. S. C. Davis et al., "Dynamic dual-tracer MRI-guided fluorescence tomography to quantify receptor density in vivo," Proc. Natl. Acad. Sci. U.S.A. 110(22), 9025-9030 (2013).

26. K. M. Tichauer et al., "In vivo quantification of tumor receptor binding potential with dual-reporter molecular imaging," Mol. Imaging Biol. 14(5), 584-592 (2012).

27. V. Chernomordik et al., "Quantitative analysis of HER2 receptors expression in vivo by NIR optical imaging," Mol. Imaging 9(4), 192-200 (2010).

28. S. Kwon et al., "Imaging dose-dependent pharmacokinetics of an RGDfluorescent dye conjugate targeted to avb3 receptor expressed in Kaposi's sarcoma," Mol. Imaging 4(2), 75-87 (2005).

29. M. Gurfinkel et al., "Quantifying molecular specificity of $\alpha_{\mathrm{v}}-\beta_{3}$ integrin-targeted optical contrast agents with dynamic optical imaging," J. Biomed. Opt. 10(3), 034019 (2005).

30. L. Zhu et al., "Dynamic PET and optical imaging and compartment modeling using a dual-labeled cyclic RGD peptide probe," Theranostics 2(8), 746-756 (2012).

31. G. Z. Ferl et al., "Derivation of a compartmental model for quantifying 64Cu-DOTA-RGD kinetics in tumor-bearing mice," J. Nucl. Med. 50(2), 250-258 (2009).

32. R. W. Holt et al., "Tomography of epidermal growth factor receptor binding to fluorescent Affibody in vivo studied with magnetic resonance guided fluorescence recovery in varying orthotopic glioma sizes," J. Biomed. Opt. 20(2), 026001 (2015).

33. N. Hamzei et al., "Comparison of kinetic models for dual-tracer receptor concentration imaging in tumors," Austin J. Biomed. Eng. 1(1) (2014).

34. R. B. Innis et al., "Consensus nomenclature for in vivo imaging of reversibly binding radioligands," J. Cereb. Blood Flow Metab. 27(9), 1533-1539 (2007).

35. J. Yin et al., "Evaluation of Tc-99 m labeled dimeric GX1 peptides for imaging of colorectal cancer vasculature," Mol. Imaging Biol. 17(5), 661-670 (2015).

36. K. Chen and X. Chen, "Positron emission tomography imaging of cancer biology: current status and future prospects," Semin. Oncol. 38(1), 70-86 (2011).

37. X. Chen, P. S. Conti, and R. A. Moats, "In vivo near-infrared fluorescence imaging of integrin $\alpha_{\mathrm{v}}-\beta_{3}$ in brain tumor xenografts," Cancer Res. 64(21), 8009-8014 (2004).

38. S. Zitzmann, V. Ehemann, and M. Schwab, "Arginine-glycine-aspartic acid (RGD)-peptide binds to both tumor and tumor-endothelial cells in vivo," Cancer Res. 62(18), 5139-5143 (2002).

Biographies for the authors are not available. 